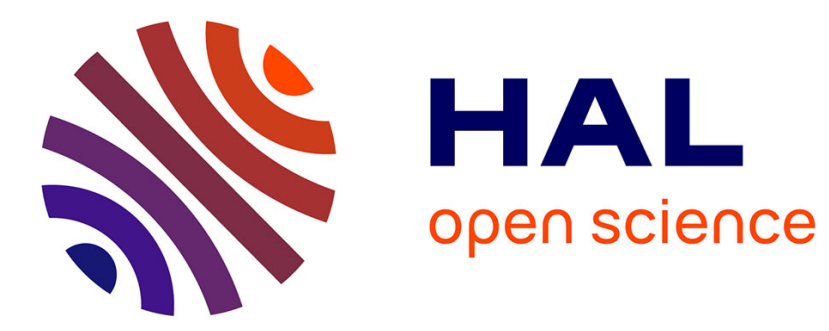

\title{
Frame decomposition of scattered fields
}

Igor Francisco Arias Lopez, Christine Letrou

\section{To cite this version:}

Igor Francisco Arias Lopez, Christine Letrou. Frame decomposition of scattered fields. ICEAA 2011: International Conference on Electromagnetics in Advanced Applications, Sep 2011, Torino, Italy. pp.1261 - 1264, 10.1109/ICEAA.2011.6046529 . hal-01306760

\section{HAL Id: hal-01306760 https://hal.science/hal-01306760}

Submitted on 25 Apr 2016

HAL is a multi-disciplinary open access archive for the deposit and dissemination of scientific research documents, whether they are published or not. The documents may come from teaching and research institutions in France or abroad, or from public or private research centers.
L'archive ouverte pluridisciplinaire HAL, est destinée au dépôt et à la diffusion de documents scientifiques de niveau recherche, publiés ou non, émanant des établissements d'enseignement et de recherche français ou étrangers, des laboratoires publics ou privés. 


\section{Frame Decomposition of Scattered Fields}

\author{
I.F. Arias Lopez*
}

Abstract - Radiated or scattered fields are represented as being radiated by a number of limited plane wave spectra obtained by subdividing the 3D spectrum into spectra defined in several planes, using a partition of unity technique. Gabor frame decomposition can be used in each spectral domain, to decompose the field spectrum into Gaussian windows radiating in the form of Gaussian beams. The summation of all beams provides a representation of fields radiated or scattered into all the directions of space. Numerical illustration and validation of this approach will be presented.

\section{INTRODUCTION}

Frame decomposition has been primarily introduced into Gaussian Beam Shooting (GBS) algorithms to perform decompositions of fields radiated by large planar apertures into a half plane, in a rigorous and stable way [1]. The ability of GBS to propagate fields at far or moderate distances, combined with paraxial beam transformations, makes it a good candidate to simulate multiple interactions in complex environments. In such contexts however, radiated or scattered fields, whatever method is used to model the scattering phenomenon, should be decomposed into Gaussian beams shooted into all directions, not only into a half space. This problem has been addressed by Complex Point Source beam expansions on spherical surfaces $[2,3]$. In this paper, we propose an alternative approach, based on frame decompositions in planes.

In many situations of practical interest for GBS application, source fields are given in the form of an antenna far field pattern. In the following, frame decomposition is thus applied in the spectral domain, starting from the knowledge of radiated or scattered far fields. The proposed formulation considers the far field in any direction as resulting from a summation of plane wave spectra defined in several planes, obtained through a classical partition of unity. It will be assumed in this paper that only a limited angular range of elevation angles need to be considered, hence the PWS partitioning

\footnotetext{
*Telecom SudParis (Lab. SAMOVAR - UMR CNRS 5157), 9 rue Charles Fourier, 91011 Evry Cedex, France.

†Telecom SudParis (Lab. SAMOVAR - UMR CNRS 5157), 9 rue Charles Fourier, 91011 Evry Cedex, France, e-mail: christine.letrou@int-evry.fr, tel.: +3316076 46 29, fax: +33160764433.
}

\author{
C. Letrou ${ }^{\dagger}$
}

is performed along one spectral variable only.

Section 2 gives a brief outline of frame decomposition and of its application in the context of a directive source radiating into a half space. Section 3 presents the spectrum partitioning approach and its combination with frame decomposition.

\section{FRAME DECOMPOSITION}

In frame based approaches, the decomposition of radiated fields into a set of Gaussian beams derives from the decomposition of a planar source distribution on a Gabor frame of Gaussian windows of two variables $[4,5]$. For sufficiently directive antennas, radiating negligible fields into a half space, one such frame decomposition of each electric field component tangent to the plane yields a representation of radiated fields in the form of a summation of Gaussian beams in the half space where the antenna radiates. In this section we review briefly the formulation of frame decomposition in this context, both in spatial and spectral domains, and the calculation of the coefficients for such decompositions. For the sake of simplicity, we shall present scalar formulations, valid for field components.

\subsection{Gabor frames in $L_{2}(\mathbb{R})$}

In the $L_{2}(\mathbb{R})$ Hilbert space, the set of Gaussian functions

$$
\begin{gathered}
\psi_{m, n}(x)=\psi(x-m \bar{x}) e^{i n \bar{k}_{x}(x-m \bar{x})},(m, n) \in \mathbb{Z}^{2} \\
\text { with } \quad \psi(x)=\sqrt{\frac{\sqrt{2}}{L}} e^{-\pi \frac{x^{2}}{L^{2}}}
\end{gathered}
$$

is a frame if and only if $\bar{x} \bar{k}_{x}=2 \pi \nu$ with $\nu<1$ (oversampling factor) [6]. $\bar{x}$ and $\bar{k}_{x}$ are respectively the spatial and spectral domain translation step.

Frames are complete sets hence any function $f \in L_{2}(\mathbb{R})$ can be expressed as a summation of weighted frame windows:

$$
f=\sum_{(m, n) \in \mathbb{Z}^{2}} a_{m, n} \psi_{m, n},(m, n) \in \mathbb{Z}^{2}
$$

with the $a_{m, n}$ complex coefficients called "frame coefficients". The Fourier transform of a function $g$, denoted $\widetilde{g}$, is defined as:

$$
\widetilde{g}\left(k_{x}\right)=\int_{-\infty}^{+\infty} g(x) e^{-i k_{x} x} d x
$$


It can be shown that the set of functions in the spectral domain $\widetilde{\psi}_{n, m},(n, m) \in \mathbb{Z}^{2}$, obtained by translations of the Gaussian function $\widetilde{\psi}$ :

$$
\widetilde{\psi}_{n, m}(x)=\widetilde{\psi}\left(k_{x}-n \bar{k}_{x}\right) e^{-i m n \bar{x} k_{x}}
$$

is also a Gabor frame in $L_{2}(\mathbb{R})$, with $\widetilde{\psi}$ a Gaussian function. Due to the relation between the Fourier transform of $\psi_{m, n}$ and $\widetilde{\psi}_{n, m}$, equation (3) yields the decomposition of $\tilde{f}$ on that frame in the spectral domain:

$$
\tilde{f}=\sum_{(n, m) \in \mathbb{Z}^{2}} a_{m, n} e^{i m n \bar{k}_{x} \bar{x}} \widetilde{\psi}_{n, m}
$$

Hence, the coefficients of the frame decomposition of a function or of its Fourier transform can be computed in either domain, spatial or spectral.

To represent field distributions in planes in the following, we shall use frames in $L_{2}\left(\mathbb{R}^{2}\right)$ defined by the product of two frames in $L_{2}(\mathbb{R})$. Unless specified, the same frame parameters will be used along both variables in $\mathbb{R}^{2}$, and the same oversampling factor will be used in spatial and spectral domains ("balanced" frames). Denoting by $(x, y)$ the variable in $\mathbb{R}^{2}$, we thus have $\bar{y}=\bar{x}=L \sqrt{\nu}$ and $\bar{k}_{y}=\bar{k}_{x}=\Omega \sqrt{\nu}$ with $\Omega=2 \pi / L$. With these conventions and by reference to (1), Gabor frame windows in $L_{2}\left(\mathbb{R}^{2}\right)$ write as:

$$
\psi_{\boldsymbol{\mu}}(x, y)=\psi_{m, n}(x) \psi_{p, q}(y), \boldsymbol{\mu}=(m, n, p, q) \in \mathbb{Z}^{4}
$$

\section{$2.2 \quad$ Frame coefficients}

The set of frame coefficients $a_{m, n} \in \mathbb{C}$ of a function $f \in L_{2}(\mathbb{R})$ is not unique and can be obtained through various algorithms. The projection of the function $f$ on the "dual frame" windows $\widehat{\psi}_{m, n},(m, n) \in \mathbb{Z}^{2}$ yields the representation with minimum energy and will be the preferred algorithm in this work:

$$
a_{m, n}=\left\langle f, \widehat{\psi}_{m, n}\right\rangle=\int_{-\infty}^{\infty} f(x) \widehat{\psi}_{m, n}^{*}(x) d x
$$

where $\widehat{\psi}_{m, n}$ is an element of the "dual frame", obtained by translation of the dual function $\widehat{\psi}$. It has been shown [5] that this function $\widehat{\psi}$ is approximately proportional to the frame Gaussian function $\psi$ :

$$
\widehat{\psi} \sim \frac{\nu}{\|\psi\|^{2}} \psi
$$

if $\nu$ is less than 0.3 (high oversampling). $\|\quad\|^{2}$ is the squared norm derived from the Hermitian product in $L^{2}(\mathbb{R})$.
The dual frame in the spectral domain is constructed by the same translations as in (5), from the dual frame window $\widehat{\widetilde{\psi}}$, which is easily obtained from the Fourier transform of the dual frame function $\widehat{\psi}$ : $\widehat{\widetilde{\psi}}=\frac{1}{2 \pi} \widetilde{\widehat{\psi}}$. If the oversampling is large enough, we have:

$$
\widehat{\widetilde{\psi}}=\frac{1}{2 \pi} \frac{\nu}{\|\psi\|^{2}} \widetilde{\psi}
$$

Finally, the $a_{m, n}$ coefficients can be computed in the spectral domain, as:

$$
\begin{aligned}
a_{m, n} & =\left\langle\widetilde{f}, \widehat{\widetilde{\psi}}_{m, n}\right\rangle \\
& =\frac{e^{-i m n \bar{k}_{x} \bar{x}}}{2 \pi} \int_{-\infty}^{\infty} \tilde{f}\left(k_{x}\right){\widetilde{\hat{\psi}_{m, n}^{*}}}^{*}\left(k_{x}\right) d k_{x}
\end{aligned}
$$

Expressions of dual frame windows and of frame coefficients for frame decomposition in $L_{2}\left(\mathbb{R}^{2}\right)$ are easily deduced from those in $L_{2}(\mathbb{R})$.

\section{PWS partitioning}

\subsection{Position of the problem}

We consider a scenario where the far field pattern of the antenna is directive in all vertical planes (elevation angle) and is not directive as a function of the azimuth angle. We take the $y$ axis as vertical, and we introduce four systems of coordinate with the same origin $O$, where the planes $\mathcal{P}_{j}\left(O, x_{j}, y\right), j=1, \ldots, 4$, are vertical with the $x_{j}$ axes oriented towards four orthogonal directions in the horizontal plane (cf Fig. 1). The respective $z_{j}$ axes are chosen so as to complete the four systems of cartesian coordinates. Spectral variables $\left(k_{x_{j}}, k_{y}\right)$ are associated to the spatial variables $\left(x_{j}, y\right)$ in the $\mathcal{P}_{j}$ plane. Plane wave spectra $\widetilde{E}_{j}, j=1, \ldots, 4$, are defined as the PWS of $E_{y}$ in the planes $\mathcal{P}_{j}$, corresponding to waves radiated in the $z_{j}>0$ half space.

Our aim is to define "partitioning" functions $\chi_{j}\left(k_{x_{j}}, k_{y}\right)$ satisfying the following conditions:

- $\chi_{j}\left(k_{x_{j}}, k_{y}\right)=0$ if $k_{x_{j}}^{2}+k_{y}^{2}>k^{2}$ with $k$ the free space wavenumber (the domain of definition of $\chi_{j}$ is included in the visible domain $z_{j}>0$ ).

- The field component $E_{y}$ radiated in the far field at a point $M$ in the half space $z_{j}>0$ is obtained by the following summation:

$$
E_{y}=\frac{-i}{\lambda r} e^{i k r} \sum_{j \in J} \cos \theta_{j} \widetilde{E}_{j}\left(k_{x_{j}}, k_{y}\right) \chi_{j}\left(k_{x_{j}}, k_{y}\right)
$$

where $r$ is the distance $O M, \cos \theta_{j}=k_{z_{j}} / k$, the wavevector with components $\left(k_{x_{j}}, k_{y}, k_{z_{j}}\right)$ components is directed towards the observation point $M$, and $j \in J$ if $z_{j}(M)>0$. 


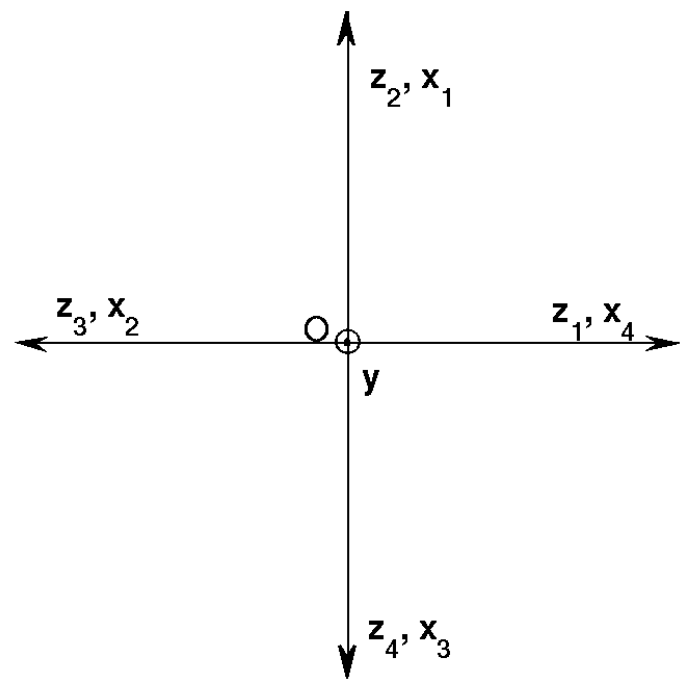

Figure 1: Coordinate systems associated to planes $\mathcal{P}_{j}\left(O, x_{j}, y\right), j=1, \ldots, 4$.

It is easily seen that $J$ is necessarily of the form $\{j, j+1\}$. To simplify notations and without lack of generality, we assume in the following that $j=1$. We also denote $\widetilde{E}_{j}^{\chi}$ the product $\widetilde{E}_{j} \chi_{j}$. The condition (12) can be rewritten as:

$$
k_{z_{1}} \widetilde{E}_{1}\left(k_{x_{1}}, k_{y}\right)=k_{z_{1}} \widetilde{E}_{1}^{\chi}\left(k_{x_{1}}, k_{y}\right)+k_{z_{2}} \widetilde{E}_{2}^{\chi}\left(k_{x_{2}}, k_{y}\right)
$$

in the "quarter" of space $k_{z_{1}}>0$ and $k_{z_{2}}>0$. Using the relation between PWS in different planes

$$
\widetilde{E}_{2}\left(k_{x_{2}}, k_{y}\right)=\frac{k_{z_{1}}}{k_{z_{2}}} \widetilde{E}_{1}\left(k_{x_{1}}, k_{y}\right)
$$

we get:

$$
\chi_{1}\left(k_{x_{1}}, k_{y}\right)+\chi_{2}\left(k_{x_{2}}, k_{y}\right)=1
$$

\subsection{Partitioning functions}

In order to minimize the spatial domain widening associated to spectral windowing, we shall use the well-known Hann window (raised cosine) which is of common use for windowing purposes related to FFT algorithms. For a given $k_{y}$ :

$$
\begin{aligned}
\chi_{1}\left(k_{x_{1}}, k_{y}\right) & =1 \quad \text { for } 0 \leq k_{x_{1}} \leq k_{L} \\
& =h\left(k_{x_{1}}, k_{y}\right) \quad \text { for } k_{L} \leq k_{x_{1}} \leq k_{L}+\delta \\
& =0 \quad \text { for } k_{L}+\delta \leq k_{x_{1}} \leq k_{h}
\end{aligned}
$$

with $k_{h}=\sqrt{k^{2}-k_{y}^{2}}$, and $\left[k_{L}, k_{L}+\delta\right]$ the "transition region". $k_{L}$ can be chosen dependant on $k_{y}$. However, if the spectrum of the antenna is directive enough in vertical planes, $k_{L}$ can be kept the same for all $k_{y}$, in as much as $k_{L}+\delta$ is smaller than the minimum value of $k_{h}$, obtained for the maximum value of $\left|k_{y}\right|$. In more general cases, the frame decomposition of the spectrum along the $k_{y}$ variable makes it possible to consider only PWS which are sufficiently localized along $k_{y}$. The Hann function in this context is taken as:

$$
h\left(k_{x_{1}}, k_{y}\right)=0.5\left(1-\cos \left[\frac{\pi}{\delta}\left(k_{x_{1}}-k_{L}+\delta\right)\right]\right)
$$

Regarding the partitioning function $\chi_{2}\left(k_{x_{2}}, k_{y}\right)$ in the "quarter" of space where $k_{z_{1}}>0$ and $k_{z_{2}}>0$, it is derived from equation (15):

$$
\begin{array}{r}
\chi_{2}\left(k_{x_{2}}, k_{y}\right)=0.5\left(1+\cos \left[\frac { \pi } { \delta } \left(\sqrt{k_{h}^{2}-k_{x_{2}}^{2}}\right.\right.\right. \\
\left.\left.\left.-k_{L}+\delta\right)\right]\right)
\end{array}
$$

in the transition region:

$$
-\sqrt{k_{h}^{2}-k_{L}^{2}} \leq k_{x_{2}} \leq-\sqrt{k_{h}^{2}-\left(k_{L}+\delta\right)^{2}}
$$

In Fig. 2 (resp. Fig. 3) are represented both $\chi_{1}\left(k_{x_{1}}, k_{y}\right)$ and $\chi_{2}\left(k_{x_{2}}, k_{y}\right)$ as functions of the spectral variable $\left(k_{x_{1}}\right.$ (resp. $\left(k_{x_{2}}\right)$, for $k_{y}=0$. It should be noted that $k_{x_{2}}=-k_{z_{1}}=-\sqrt{k^{2}-k_{x_{1}}^{2}}$.

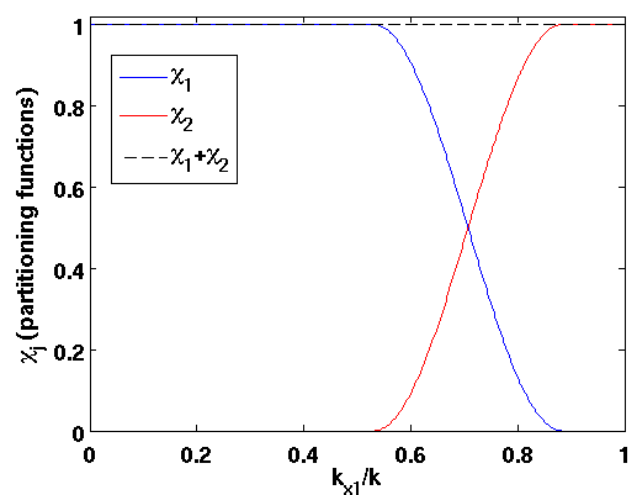

Figure 2: Partitioning functions $\chi_{1}\left(k_{x_{1}}, 0\right)$ and $\chi_{2}\left(k_{x_{2}}\left(k_{x_{1}}\right), 0\right) . \delta=k /(2 \sqrt{2}), k_{L}=k / \sqrt{2}-\delta / 2$.

\section{CONCLUSION}

Frame decomposition can be performed in each of the spectral domains described by the $\left(k_{x_{j}}, k_{y}\right)$ variables, for the field radiated by the corresponding windowed PWS $\widetilde{E}_{j}^{\chi}$ into the half space $k_{z_{j}}>0$. It is expected that this technique will allow for GBS into all regions of space, and will also avoid launching highly tilted beams, whose paraxial formulas are known to be inaccurate. The price to pay is an increase in the number of spatially translated Gaussian windows to be taken into account in each of the frame decompositions. Numerical examples will be presented to validate this approach and quantify the number of windows in decompositions. 


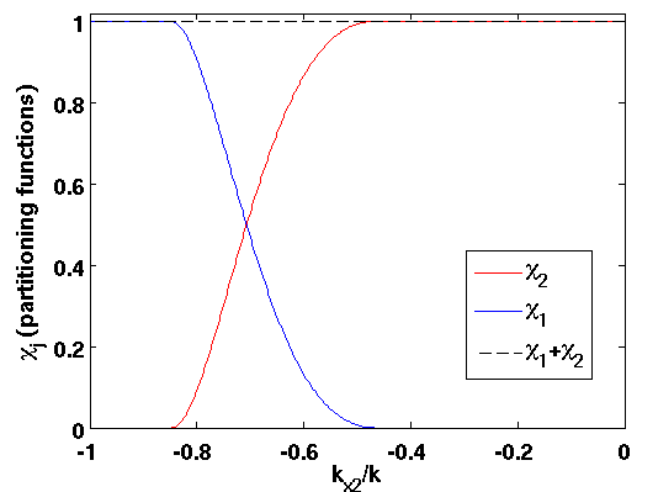

Figure 3: Partitioning functions $\chi_{1}\left(k_{x_{1}}\left(k_{x_{2}}\right), 0\right)$ and $\chi_{2}\left(k_{x_{2}}, 0\right) . \delta=k /(2 \sqrt{2}), k_{L}=k / \sqrt{2}-\delta / 2$.

\section{References}

[1] D. Lugara and C. Letrou, "Alternative to Gabor's representation of plane aperture radiation," Electron. Lett., vol. 34, no. 24, pp. 22862287, Nov. 1998.

[2] K. Tap, P. Pathak, and R. Burkholder, "An exact CSP beam representation for EM wave radiation," in International Conference on Electromagnetics in Advanced Applications (ICEAA '07), Torino, Italy, Sept. 2007, pp. 75-78.

[3] G. Carli, E. Martini, and S. Maci, "Space decomposition method by using complex source expansion," in IEEE AP-S Intl. Symp., San Diego, CA, July 2008, pp. 1-4.

[4] D. Lugara, C. Letrou, A. Shlivinski, E. Heyman, and A. Boag, "Frame-based gaussian beam summation method: Theory and application," Radio Science, vol. 38, no. 2, Apr. 2003.

[5] A. Shlivinski, E. Heyman, A. Boag, and C. Letrou, "A phase-space beam summation formulation for ultrawide-band radiation," IEEE Trans. Antennas Propagat., vol. 52, no. 8, pp. 2042-2056, 2004.

[6] I. Daubechies, Ten lectures on wavelets, ser. CBMS-NSF Regional Conference Series in Applied Mathematics. Philadelphia: SIAM Press, 1992, vol. 61 . 